\title{
ISOTOPIC RATIOS IN COMETS
}

\author{
V. VANYSEK \\ Astronomical Institute of \\ University Erlangen - Nuernberg \\ Dr.-Remeis-Observatory \\ 8600 Bamberg, F.R.G.
}

\begin{abstract}
The isotopic abundances depend on the universal evolution of elements and on the individual history of particular objects. Since it is believed that unprocessed material of the solar nebula is preserved in comets, the data concerning the abundance of stable isotopes in these primitive bodies are of some importance in the cosmological context. The present status of this problem is reviewed. The reliability of results for nuclear species with cosmological and cosmogonical implications, such as D/H, C 12/13, N 14/15, $\mathrm{O} 16 / 18$, and $\mathrm{Mg} 24 / 25 / 26$, is discussed. Significant variation is found for the isotopic abundance of carbon, depending upon which carbon reservoir is sampled. Deuterium is probably enhanced relative to the interstellar ratio. For other isotopes, the ratios are close to those of the terrestrial data. The tendency of the $\mathrm{D} / \mathrm{H}$ ratio to be at higher values indicates a low temperature in the environment of the comet's formation, and, together with similar effects in the outer planets, suggests that there were two different primordial reservoirs of deuterium in the solar system. The ${ }^{12} \mathrm{C} /{ }^{13} \mathrm{C}$ ratio inferred from in situ mass spectrometry of the dust, as well as from the ground-based optical spectra of the Swan band, tends to be approximately equal to the average terrestrial ratio (89) or larger. Recent results obtained from the $\mathrm{CN}$ band provide a significantly lower value (about 65 ), which corresponds to the carbon isotopic ratio in the diffuse interstellar clouds. The enhancement of deuterium and the possible differences of the carbon isotopic ratio in different species and refractory material are indicative of chemical fractionation processes in the protosolar nebula.
\end{abstract}

\section{Introduction}

The observed properties of comets indicate that cometary nuclei are more or less fragile, low-density bodies in which pristine material left over from the presolar nebula may be confined. However, this statement should be regarded as a research concept rather than as a consensus concerning the structure and origin of comets. There is no evidence that the cometary nuclei retain exactly the molecular composition inherited from the presolar molecular cloud or even from interstellar diffuse matter. Nevertheless, in terms of the molecular composition, there seems to be a direct relation between cometary material and 
the material of dense, cool interstellar clouds. Thus, in our concept, it is assumed that comets appear to be relatively or absolutely unaltered condensates that contain key information about processes that took place in a cloud preceding the early stages of the solar nebula. From this point of view, data concerning the isotopic ratios in comets could be most interesting and significant. The isotopic abundances, which depend on the universal evolution of elements and on the individual history of the particular objects, can be used as one of the crucial tests for cosmological models, theories of the chemical evolution of our galaxy, and theories of solar system evolution. Although data on the stable isotopes in comets are known for only a few elements for a few objects, and are known with only low accuracy, the progress in this field is significant, and the isotopic abundances in comets certainly will be a very important research area in the near future.

The cometary nucleus contains volatile and refractory material, i.e., nonuniformly mixed constituents. The cometary dust should be regarded as material with an evolutionary history that entirely precedes the development of planetesimals and even the formation of the solar nebula. The dust grains growing in the interstellar environment keep their chemical composition virtually unchanged, since only minor variations of the grain temperature are expected during the formation of the cometary nucleus. Consequently, the isotopic abundances, like the chemical composition, in the refractory component of cometary dust are inherited from those in the interstellar matter. On the other hand, the condensable volatile constituents in the ice component have most likely been subject to modification and redistribution of particular chemical compounds. Therefore, the isotopic ratios in dust grains may be different from those in the cometary ice. The abundances of various chemical species in interstellar space, as well as in dense clouds, are predominantly controlled by the reaction rates appropriate for those environments. Under the conditions generally prevailing in cool interstellar environments, isotopic fractionation is an important process that may redistribute the isotopic ratios in cometary material. Therefore, the isotopic data derived from the evaporated molecular compounds cannot be considered as being representative of the cometary dust and vice versa.

It must be noted, however, that the data obtained either by a standard ground-based method or by "in situ" mass spectrometry do not allow the determination of the small differences in isotopic ratios that laboratory methods can commonly detect in meteorites. Moreover, the mass spectrometry of dust is, to some extent, hampered by contamination of the atomic vapor released from the evaporated dust grains by not completely disintegrated molecular species. In particular, a "confusion problem" may result from various mass ambiguities $\left({ }^{13} \mathrm{C}\right.$ vs. ${ }^{12} \mathrm{C}^{1} \mathrm{H}$, etc.). Thus, only isotopic ratios that deviate by a factor of two or greater from the expected isotopic "normal" value can be considered to be "significantly anomalous."

Table 1 summarizes data about the ratios of some stable isotopes derived for various astronomical objects. The data presented either have been averaged or are only ranges of the observed values. Further information about more complete data may be found in the papers cited in the table's footnotes.

\section{Deuterium}

Since almost the entire deuterium inventory was most likely synthesized in the early universe, the deuterium-to-hydrogen ratio, defined here as $\mathrm{D} / \mathrm{H}$, is one of the crucial 
Table 1. Ratios of Some Stable Isotopes

Species Solar System ${ }^{a} \quad$ Local ISM $^{\mathbf{b}} \quad$ Comets

$\begin{array}{cccc}\mathrm{H} / \mathrm{D} & 2 \times 10^{-5} & 1.5 \times 10^{-5} \mathrm{c} & \geq 5 \times 10^{-4} \mathrm{~d}, \mathrm{e} \\ \mathrm{H} / \mathrm{D} & - & 1 \times 10^{-4} \text { to } 1 \times 10^{-2} \mathrm{f} & - \\ 12 \mathrm{C} / 13 \mathrm{C} & 89 & 43 \pm 4 \mathrm{~g} & 65 \pm 9 \mathrm{e}, \mathrm{h} \\ 12 \mathrm{C} / 13 \mathrm{C} & - & 65 \pm 20^{\mathrm{i}} & 70 \text { to } 130 \mathrm{j} \\ 12 \mathrm{C} / 13 \mathrm{C} & - & 12 \text { to } 110^{\mathrm{f}} & \ll 100 \mathrm{e}, \mathrm{k}, 1 \\ 14 \mathrm{~N} / 15 \mathrm{~N} & 270 & \approx 400^{\mathrm{i}} & >200 ? \mathrm{~m} \\ 16 \mathrm{O} / 18 \mathrm{O} & 490 & \approx 400^{\mathrm{i}} & <450 \mathrm{e}, \mathrm{n} \\ 24 \mathrm{Mg} / 25 \mathrm{Mg} & 7.8 & - & \text { variable e, } \mathrm{e}, \mathrm{o} \\ 25 \mathrm{Mg} / 26 \mathrm{Mg} & 0.9 & - & <2 \mathrm{e}, \mathrm{o} \\ 32 \mathrm{~S} / 34 \mathrm{~S} & 22.6 & - & 22 \mathrm{e}, \mathrm{o} \\ 56 \mathrm{Fe} / 54 \mathrm{Fe} & 15.8 & - & 15 \mathrm{e}, \mathrm{o}\end{array}$

a These values are compiled. For more details and references, see an earlier review paper by Encrenaz (1984).

b ISM = interstellar matter.

c Average value derived from ultraviolet (UV) spectra (Pasachoff and Vidal-Madjar (1989)).

d Mass spectrometry in situ by Eberhardt et al. (1987).

e Data for P/Halley.

f Range of observed values in dense interstellar clouds.

g Obtained from visual spectra (Hawkins and Jura (1988)).

h From spectra of the CN band (Wyckoff et al. (1989)).

i Radio astronomical data. For original references, see Combes et al. (1980), Penzias (1983), Gerin et al. (1984), and Bastien et al. (1984).

j Range of values for several comets from the Swan band. For data discussion and references, see Danks, Lambert, and Arpigny (1974), Vanysek and Rahe (1978), and Lambert and Danks (1983).

$\mathrm{k}$ The data for dust particles obtained by in situ mass spectrometry. Values are spread in the range from 20 to $1,000(!)$, with a maximum around 100 . For references, see Jessberger (1990), in this volume.

1 Data partly hampered either by the instrumental artifacts or by mass ambiguities.

$m$ Estimated possible lower limit from visual spectra (Wyckoff et al. (1989)).

n From Eberhardt et al. (1987).

0 The data for dust particles obtained by in situ mass spectrometry. Values are spread in the range from 20 to 1,000 (!), with a maximum around 100 . For references, see Jessberger (1990), in this volume. See also Solc et al. (1987). 
parameters for the estimation of the baryonic density parameter of the universe. Determination of the $\mathrm{D} / \mathrm{H}$ value in various astronomical objects, including comets, is one of the most important goals of the contemporary observational astrophysics and space research.

Although hydrogen is one of the most abundant species in the cometary atmosphere, contemporary spectroscopic techniques have not been able to provide acceptable data on deuterium. The amount of deuterium can, in principle, be inferred from the emission (or absorption) line in the wing of the hydrogen Lyman $\alpha$ line. However, this line is extremely strong in comparison with the expected intensity of the adjacent deuterium feature, and this fact is the main problem in the determination of the $\mathrm{D} / \mathrm{H}$ ratio from atomic spectra. Thus, as for other objects, the $\mathrm{D} / \mathrm{H}$ ratio must be derived-more or less straightforwardly-from deuterated molecules. Since water contains almost all of the hydrogen content in comets, the determination of the $\mathrm{HDO} / \mathrm{H}_{2} \mathrm{O}$ or the $\mathrm{OD} / \mathrm{OH}$ ratio seems to be the most reliable method to obtain information about the $\mathrm{D} / \mathrm{H}$ ratio in these objects. However, another method, not yet applied, was proposed by Singh and Dalgarno (1987), based on the determination of the $\mathrm{CD} / \mathrm{CH}$ ratio. The absorption lines at 314.209 and $314.32 \mathrm{~nm}$ of these radicals may be detectable in the coma if a bright star can be used as a background source.

Because the basic structure (i.e., the electronic, vibrational, and rotational structure) of OD is identical to that of $\mathrm{OH}, \mathrm{A}^{\prime} \mathrm{Hearn}$ et al. (1985) applied the same program for the theoretical determination of $\mathrm{OH}$ as they did for that of the $\mathrm{OD}$ fluorescence band intensity. From the observed intensity of $\mathrm{OH}$ in International Ultraviolet Explorer (IUE) spectra of comets $1978 \mathrm{XV}, 1979 \mathrm{X}, 1980 \mathrm{X}, 1982 \mathrm{VI}$, and $1983 \mathrm{~d}$, A'Hearn et al. estimated the threshold intensity of OD (0-0), and the upper limit of the D/H inferred from the best data yielded a value of 0.007 . However, the IUE data for $\mathrm{OD} / \mathrm{OH}$ only indicate the possibility that the deuterium could be enriched in cometary water by a factor of 100 or more relative to the upper limit of $\mathrm{D} / \mathrm{H}=5 \times 10^{-5}$ derived for diffuse interstellar matter (Vidal-Madjar (1983); Pasachoff and Vidal-Madjar (1989)).

The $\mathrm{D} / \mathrm{H}$ ratio derived in situ for $\mathrm{HDO} / \mathrm{H}_{2} \mathrm{O}$ in $\mathrm{P} /$ Halley by Eberhardt et al. (1987) from the mass spectrometry data provided by the Giotto space probe is in the range of $5 \times 10^{-5}$ to $5 \times 10^{-4}$. The average of these values does not differ significantly from that found for the Titan atmosphere and outer planets (see Lutz et al. (1983), de Bergh et al. (1986), and H.W. Smith et al. (1989)) and is almost the same as that in the Standard Mean Ocean Water (SMOW). This indicates that the cometary volatile material is enriched in deuterium by a factor of about 10 relative to the $\mathrm{D} / \mathrm{H}$ ratio assumed for the protoplanetary nebula from the studies of ${ }^{3} \mathrm{He}$ in meteorites (see Geiss and Reeves (1981) and references therein). There is the problem of mass ambiguities in the mass spectrometric data caused by $\mathrm{NH}_{4}^{+}$or $\mathrm{H}_{3} \mathrm{O}^{+}$, but this effect can be estimated, in principle, from the abundance of $\mathrm{H}_{2} \mathrm{DO}^{+}$relative to the other species.

Although an update of observational results provides only meager data concerning the value of $\mathrm{D} / \mathrm{H}$ in comets, the tendency for deuterium enhancement in these bodies seems to be real and is in agreement with predictions published some years ago (Ip (1985) and Vanysek and Vanysek (1985)).

Many processes in interstellar clouds-and, presumably, also in the solar nebulamay lead to isotopic fractionation in molecules at low temperatures. Thus, the relative abundances of isotopes, including the $\mathrm{D} / \mathrm{H}$ ratio, may deviate from their primordial values. The estimation of deuterium abundance in the solar nebula has been the topic of many 
studies (Geiss and Reeves (1972), Black (1973), Robert et al. (1979), Hubbart and McFarlane (1980), Gautier and Owen (1983), and Grispoon and Lewis (1988)).

The chemical reactions at low temperatures in interstellar matter-and consequently also in the solar nebula - may have led to enrichment of deuterium in condensable species. Since these processes are highly temperature-dependent (for a discussion, see D. Smith (1987)), the observed $\mathrm{D} / \mathrm{H}$ ratio might, in principle, be used as a temperature indicator of the environment where the comets or protoplanetary bodies were formed.

Radio astronomical observations of deuterated species in dense interstellar clouds provide very strong evidence that chemical fractionation is the only process that is obviously responsible for the very high $\mathrm{D} / \mathrm{H}$ ratio in many molecules. The main channels for deuteration in interstellar molecules are ion-neutral reactions with large reaction rates. Besides these, selective photodissociation is most likely a very efficient fractionation mechanism also. The well-known example for a typical ion-neutral reaction is

$$
\mathrm{D}^{+}+\mathrm{H}_{2} \Leftrightarrow \mathrm{H}^{+}+\mathrm{HD}+\Delta E
$$

owing to the difference in the zero-point energies of molecular hydrogen and HD. This reaction is strongly exothermic to the right, and the backward reaction occurs at a significantly lower rate at low temperatures. The ratio of rate coefficients $k_{\mathrm{f}} / k_{\mathrm{b}}$ for forward/ backward reactions is

$$
k_{\mathrm{f}} / k_{\mathrm{b}}=A / \exp (B / T)
$$

For most relevant reactions, the rate constant $A \approx 10^{-9} \mathrm{~cm}^{3} \mathrm{~s}^{-1}$, and the value of $B=-\Delta E / k>100 \mathrm{~K}$ (in reaction (1), $B=460 \mathrm{~K}$ ). Therefore, much of the deuterium in dense, cool interstellar clouds is contained in $\mathrm{HD}$. Some other reactions that produce deuterated molecules are, for example,

$$
\mathrm{H}_{3}^{+}+\mathrm{HD} \Leftrightarrow \mathrm{H}_{2} \mathrm{D}^{+}+\mathrm{H}_{2}
$$

or

$$
\mathrm{HCO}^{+}+\mathrm{D} \Leftrightarrow \mathrm{DCO}^{+}+\mathrm{H}
$$

These and related subsequent reactions are the main channels for enhancement of deuterium (relative to hydrogen) in interstellar molecules (for references, see D. Smith (1987), Brown and Rice (1986), Herbst (1988), and Millar et al. (1989)). A set of quasi-time-dependent models for deuteration of $\mathrm{HD}, \mathrm{HDO}, \mathrm{DCN}$, and other species were computed by Brown and Rice (1986). For models compatible with dark clouds such as TMC-1, it was found that the time required for saturation of all considered species by deuterium was about $3 \times 10^{5}$ years, with a $\mathrm{D} / \mathrm{H}$ enrichment by a factor of 100 or more relative to the average $\mathrm{D} / \mathrm{H}$ ratio in diffuse ISM. These results are consistent with the observed high ratio of deuterated molecules in dense, cool clouds.

The time-dependent models of the gas-grain-deuterium chemistry in a dense, quiescent interstellar cloud, including accretion on the dust grains, were recently computed by Brown and Millar (1989a, 1989b). They found that as accretion begins to dominate the chemistry, the destruction rate of many radicals is reduced. This is particularly the case for 
deuterated species. The grain surface reactions may produce large deuterium enhancements in the solid phases of deuterated water, ammonia, and methane.

The neutral-neutral reactions may also lead to an enrichment of deuterium in molecular species, but owing to low reaction rates, these processes are slow and less efficient than the ion-neutral reactions. Nevertheless, Beer and Taylor (1973) and Owen et al. (1986) suggested that exchange reactions that may be summarized as

$$
\mathrm{CH}_{4}+\mathrm{HD} \Leftrightarrow \mathrm{CH}_{3} \mathrm{D}+\mathrm{H}_{2}
$$

are responsible for the enrichment of the $\mathrm{D} / \mathrm{H}$ ratio in the atmosphere of Jupiter and Saturn.

Photodissociation is another possible way to produce enhancement of D in some species, namely, in the polycyclic aromatic hydrocarbons (PAH), as was pointed out by Allamandola et al. (1987). The C-D bond zero-point energy is about $30 \%$ lower than that of the $\mathrm{C}-\mathrm{H}$ bond, so the $\mathrm{C}-\mathrm{H}$ bond will be broken more easily. This process is proposed as a mechanism that may lead to the deuterium enrichment in carbonaceous meteorites.

If comets formed in a dense environment at low temperatures, and the fractionation occurs before the cometesimals' formation, then the deuterated species with higher $\mathrm{D} / \mathrm{H}$ ratios might condense on dust particles before they are confined in the cometary nuclei. Vanysek and Vanysek (1985) suggested that ion-neutral reactions may lead to an enhancement of the $\mathrm{D} / \mathrm{H}$ ratio in comets by at least a factor of 10 . Ip (1985), who suggested that the surface reactions proposed by Tielens (1983) are the main channels for the deuteration of water in cometary volatiles, predicted an even larger $\mathrm{D} / \mathrm{H}$ enhancement (up to a factor of 1,000 ). Although these predictions are based on different assumptions regarding the dominant reactions, the results indicate that there is a tendency toward a substantial enhancement of $\mathrm{D} / \mathrm{H}$ in cometary ice.

Since the deuteration process in molecular species is virtually terminated by the burial of molecules in grains or ices (Geiss (1988)), the enrichment of condensates or organic refractories by deuterium depends on the time-scales of the processes involved. However, the condensable molecular species in dense, cool interstellar clouds are most likely highly deuterated. Therefore, the abundance of $\mathrm{D}$ may be substantially enhanced in cometary ices, even if the formation of cometary nuclei was a relatively fast process in comparison with the time-scale of the presolar nebula formation. Recently, Lutz et al. (1989) estimated the $\mathrm{D} / \mathrm{H}$ ratio that may have been present in primitive ices in the protosolar nebula. Lutz et al. developed simple, but limiting, models that constrain the amount of deuterated volatiles diluted in the original hydrogen envelopes of giant planets. These models suggest that the $\mathrm{D} / \mathrm{H}$ ratio in primitive ices was in the range of $10^{-4}$ to $10^{-3}$. These values indicate an enrichment of $\mathrm{D} / \mathrm{H}$ by a factor of 10 to 100 or more relative to the mean deuterium-to-hydrogen value found in diffuse interstellar matter.

Indirect support for the assumption that the deuterium in comets could be enhanced follows from results concerning the isotopic ratios in primitive meteorites. The chemical properties of the carbonaceous phase in the interplanetary dust particles and meteorites are indicative of an interstellar component. It was found that organic polymer fractions of $\mathrm{C}$ chondrites are strongly enriched in deuterium and that the derived $\mathrm{D} / \mathrm{H}$ ratio is 30 times higher than the mean galactic value of this ratio and is significantly larger than the SMOW value (for discussion, see Anders (1987) or McKeenan et al. (1985)). These D/H ratios correlate with $\mathrm{H}$ and $\mathrm{C}$ concentrations in interplanetary dust samples, which implies that the deuterium-rich component is identical with the hydrogenated organic phase that was found 
in bulk samples and acid residues of certain meteorites. Yang and Epstein (1983) attributed deuterium enrichment in meteorites to a carrier formed in the gas phase by the ion-molecule reactions at a temperature below $120 \mathrm{~K}$, i.e., by the same mechanism that is thought to be responsible for the deuterium enrichments in interstellar molecules.

Assuming that the enhancement of the D/H in comets is real, then this enhancement, together with similar results obtained for the outer planets, as is demonstrated in Figure 1, gives us evidence of two distinct reservoirs of deuterium in our solar system, as proposed by Owen et al. (1986). One of these reservoirs contains deuterium with an unaltered abundance and a value of $\mathrm{D} / \mathrm{H}$ that is identical to that of $\mathrm{D} / \mathrm{H}$ in the primordial solar nebula. The second deuterium reservoir is characterized by enhancement of the $\mathrm{D} / \mathrm{H}$ ratio. The enrichment of this reservoir by deuterium occurred most probably before the solar system was formed and definitely before cometesimals were formed.

\section{Stable Carbon Isotopes in Comets}

The only isotopic ratio that has been determined with an acceptable accuracy in more than one comet is the ratio of stable carbon isotopes 12 and 13. Available data are summarized in Table 2. Most of these data were derived from spectrophotometry of the Swan ${ }^{12} \mathrm{C}^{12} \mathrm{C}$ and ${ }^{12} \mathrm{C}^{13} \mathrm{C} \Delta \mathrm{v}=+1$ bands. Only in the case of Comet West (Lambert and Danks (1983)) were the $\Delta v=0$ bands used. Derivation of the carbon isotopic ratio from the violet $\mathrm{CN}$ band was successfully carried out in the case of Comet Halley (Wyckoff $e t$ al. (1989)). Also, in situ measurements by mass spectrometry are available for Comet Halley. However, they provide information only about the relative intensity of signals attributed to the ions with atomic masses 12 and 13 a.m.u., which may be assigned, although very cautiously, to the signals of carbon 12 and 13 ions. The differences in isotopic ratios obtained by the different methods constitute a problem. The data obtained from $\mathrm{C}_{2}$ bands tend to be higher than, but within the error of, the solar system value (see Table 1). On the other hand, very precise measurements in the high-resolution spectra of Comet Halley by Wyckoff et al. (1989) of isotopically shifted ${ }^{13} \mathrm{CN}$ rotational lines in the $\mathrm{CN}$ $\mathrm{B}^{2} \Sigma^{+}-\mathrm{X}^{2} \Sigma^{+}(0,0)$ band provide ${ }^{12} \mathrm{C} /{ }^{13} \mathrm{C}=65 \pm 9$, which represents either an enrichment of carbon 13 or a depletion of carbon 12 by a factor of 1.3 relative to the solar system average value. The applied method of the $\mathrm{CN}$ band analysis was discussed by Zucconi and Festou (1986).

The results obtained from the PUMA experiment, i.e., from mass spectroscopy, discussed by Solc et al. (1987), Jessberger et al. (1986), and Jessberger (1990), exhibit very large variations in the ion signal of the $12 / 13$ ratio in the range from 10 to 1,000 (in some cases, up to $4,000 !$ ). The serious problem in the interpretation of the impact mass spectrometry of the cometary dust is that not all data can be directly interpreted as being ${ }^{12} \mathrm{C} /{ }^{13} \mathrm{C}$ ratios. In the range of low values for the ion ratios, the mass at 13 a.m.u. can be due in part to ${ }^{12} \mathrm{CH}$ contamination. On the other hand, a very high ratio of $12 / 13$ a.m.u. masses $>200$ may be the result of an instrumental artifact. Nevertheless, there is a statistically significant grouping of signals in the best quality spectra around the value $12 / 13$ a.m.u. $=100$, which can tentatively be interpreted as being a real isotopic ratio that tends to be equal to the solar system ratio. Another grouping, around the value of $12 / 13$ a.m.u. $\leq 20$, cannot be seriously interpreted, because there is no unbiased method that can discriminate between true and false ${ }^{12} \mathrm{C} / 13 \mathrm{C}$ signals. 


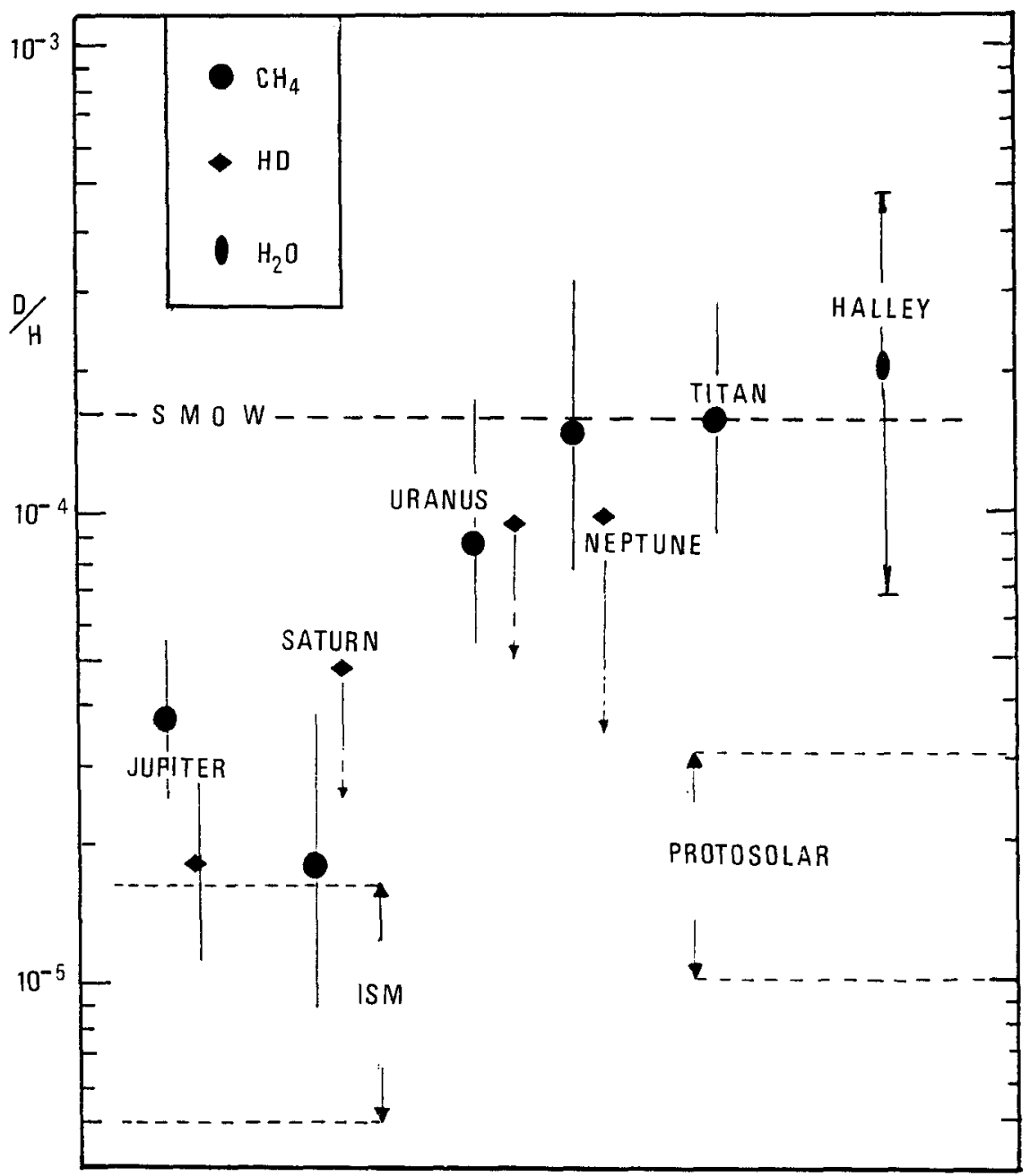

Figure 1. The deuterium-to-hydrogen ratio in various objects in the solar system compared with the range of observed values of $\mathrm{D} / \mathrm{H}$ ratios in diffuse interstellar matter (ISM) and in the solar nebula (PROTOSOLAR), and with the value for Standard Mean Ocean Water (SMOW). For references about the D/H ratio in the ISM, see Vidal-Madjar (1983) or Pasachoff and Vidal-Madjar (1989). The range of this ratio in the solar nebula was assumed from studies of ${ }^{3} \mathrm{He}$ in meteorites and based on various theoretical assumptions (see further references in the text). The $\mathrm{D} / \mathrm{H}$ ratio in the atmosphere of Comet Halley was derived from in situ measurements of relative abundance of HDO (Eberhardt et al. (1987)). The ratios from deuterated methane were derived for Uranus (de Bergh et al. (1986)) and Neptune (de Bergh et al. (1989)). Deuterated molecular hydrogen was used for the determination of the D/H ratio by H.W. Smith et al. (1989). 
Table 2. Carbon Isotopic Abundance Ratio in Comets

\begin{tabular}{lccl}
\hline \multicolumn{1}{c}{ Comet } & ${ }^{12} \mathrm{C} /{ }^{13} \mathrm{C}$ & $\begin{array}{c}\text { Mea- } \\
\text { sured a }\end{array}$ & \multicolumn{1}{c}{ Reference } \\
\hline Ikeya 1963 I & $70 \pm 15$ & $\mathrm{C}_{2}$ & $\begin{array}{l}\text { Stawikowski and Greenstein } \\
(1964)\end{array}$ \\
Tago-Sato-Kosaka 1969 IX & $100 \pm 20$ & $\mathrm{C}_{2}$ & Owen (1973) \\
Kohoutek 1973 XII & $115_{-20}^{+30}$ & $\mathrm{C}_{2}$ & Danks et al. (1974) \\
Kobayashi-Berger-Milon 1975 IX & $135_{-45}^{+65}$ & $\mathrm{C}_{2}$ & Danks et al. (1974) \\
Halley 1986 III & $100_{-30}^{+20}$ & $\mathrm{C}_{2}$ & Vanysek (1977) \\
\hline
\end{tabular}

a Measurements were made from $\mathrm{C}_{2} \Delta \mathrm{v}=+1$ bands of the Swan system and from the violet $\mathrm{CN}$ band (see text).

Determination of the carbon isotopic ratio based on classical spectroscopy of the Swan band system also poses several problems. Although the population in various levels of ${ }^{12} \mathrm{C}^{13} \mathrm{C}$ and of ${ }^{12} \mathrm{C}^{12} \mathrm{C}$ is identical and the isotopic ratio can be derived directly from the intensity ratio, there are two sources of possible systematic errors: one is caused by $\mathrm{NH}_{2}$ emission lines at 474.46 and $474.43 \mathrm{~nm}$, which partly blend the isotopically shifted $\Delta v=+1$ band; the other is the uncertainty of the continuum level in the blue wing of this band. The data derived from spectra with a strong continuum may easily lead to overestimation of the continuum intensity and consequently to overestimation of the ${ }^{12} \mathrm{C} / 13 \mathrm{C}$ ratio. The theoretical profile of the ${ }^{12} \mathrm{C}^{13} \mathrm{C} \Delta \mathrm{v}=0$ band calculated by Krishna Swamy (1987) and compared with observed profiles obtained by Lambert and Danks (1983) for Comet West shows that some features can fit a terrestrial isotopic ratio, but that a significantly lower ratio can be estimated from other features.

There are obvious uncertainties, possibly due to blends or systematical errors, about values derived from the Swan bands, but, in principle, the differences in isotopic data are not surprising at all. Since cometary material can be regarded as being almost an unprocessed relic either of the protosolar nebula or of some condensates in a dense interstellar cloud, the ${ }^{12} \mathrm{C} /{ }^{13} \mathrm{C}$ ratio in comets may exhibit large variations in the volatile and refractory species if the entire cometary material was not uniformly affected by some mixing process. There are no reasonable arguments suggesting that the same isotopic abun- 
dances prevail in solid particles as in frozen volatiles. The cometary dust should be regarded as material with its own evolutionary history that entirely precedes the development of the solar nebula.

The terrestrial ${ }^{12} \mathrm{C} /{ }^{13} \mathrm{C}$ ratio of 89 stems from the time when the solar system was formed out of the galactic gas and dense dust clouds, while the ratio in the interstellar medium reflects the present conditions. The observed interstellar ratio is still uncertain in the diffuse ISM, but seems to be about uniform and significantly below the terrestrial value (Hawkins and Jura (1987)).

Observations of molecular transitions mostly in the radio spectral ranges provide data about ${ }^{12} \mathrm{C} /{ }^{13} \mathrm{C}$ isotope ratios in dense clouds in both nearby and distant galactic regions. The average values for the carbon isotope ratio derived from $\mathrm{CO}$ and formaldehyde in several local clouds are almost identical with the terrestrial ratio, and only the Orion A region and a few other clouds exhibit values below 44, which corresponds with the lower limit predicted by some models of the chemical evolution of our galaxy (Audouze $e t$ al. (1977)). A different situation seems to be present in the diffuse ISM in the neighborhood of the Sun. From the optical observation of $\mathrm{CH}^{+}$in the direction of five bright stars, Hawkins and Jura (1987) found a weighted mean ratio of ${ }^{12} \mathrm{C} /{ }^{13} \mathrm{C}=43 \pm 4$. They also found that the ${ }^{12} \mathrm{C} /{ }^{13} \mathrm{CH}^{+}$abundance ratios along different lines of sight are identical within $12 \%$. These results favor chemical evolution models of our galaxy, based on the assumed gradual enrichment of the ISM by secondary (i.e., heavy) elements from the material ejected from stars. The carbon isotopic ratios in the ejecta from massive stars (if $\mathrm{H}$ burning is assumed) are about 3 to 7 . Ejecta from classical novae have predicted and observed values for these isotopes in the range of 0.5 to 2 . Main contributors to the ${ }^{13} \mathrm{C}$ enrichment in the interstellar matter are most likely intermediate-mass stars of about 3 to 8 solar masses (Sarmiento and Piembert (1985)).

The low value of the carbon isotopic ratio in local interstellar matter and in cometary $\mathrm{CN}$ led to the revival of speculations about the interstellar origin of comets. The simplified and straightforward approach to this problem would be an assumption that comets were formed relatively recently, in interstellar matter enriched with ${ }^{13} \mathrm{C}$, before they were captured by the solar system. Such an interpretation might seem attractive at first sight, but it is unacceptable from several points of view. Apart from impossible dynamical constraints, one must consider the possible (even if inconclusive) variation in the ratio values inferred from observations of $\mathrm{CN}$ and $\mathrm{C}_{2}$ molecules and from in situ measurements of the cometary dust.

The isotopic ratios derived for the observed molecules in the cometary atmosphere are an inherent property from the precursors of these species. In the case of $\mathrm{CN}$, the parents are $\mathrm{HCN}$ and perhaps to some extent $\mathrm{CHON}$ particles. If the precursors of the volatile compounds condensed in interstellar matter of a ${ }^{13} \mathrm{C}$-rich environment, the enhanced ratio of carbon isotopes should be detectable in all carbon-bearing molecules. However, available data exhibit significant differences between results derived from $\mathrm{CN}$ and $\mathrm{C}_{2}$ bands. If these differences are not merely an effect of systematic errors, one must consider an isotopic fractionation process.

The fractionation of carbon isotopes can occur via the well-known ion-molecule reaction

$$
{ }^{13} \mathrm{C}^{+}+{ }^{12} \mathrm{CO} \Leftrightarrow{ }^{12} \mathrm{C}^{+}+{ }^{13} \mathrm{CO}+\Delta E
$$


The difference in zero energies of the isotopic variants is $\Delta E / \mathrm{k}=35 \mathrm{~K}$; thus, the fractionation process is effective in cool environments. This reaction was proposed as a key channel responsible for the enhanced ${ }^{13} \mathrm{C}$ abundance in $\mathrm{CO}$ observed in some dense interstellar clouds (D. Smith (1987) and references therein). A consequence of this process is an anti-correlation of the carbon isotope ratio in molecules unrelated to $\mathrm{CO}$. In other words, molecules with ${ }^{13} \mathrm{CO}$ precursors may be enriched in carbon 13 and depleted in carbon 12, while other molecules exhibit an opposite tendency. A very detailed study of various models by Langer et al . $(1984,1989)$ shows that ${ }^{13} \mathrm{CO}$ should be slightly enhanced with respect to $12 \mathrm{CO}$, but the depletion of carbon 13 and, consequently, enhancement in carbon 12 in other, less abundant carbon-bearing species - for instance, in formaldehydewill be significant. However, the comparison of the computed ranges of carbon isotope ratios with the observational data indicates that the predicted enhancement of carbon 12 in formaldehyde is about a factor of 2.5 larger than the observed values.

This discrepancy can be explained by the self-shielding effect. Since the optical thickness of ${ }^{12} \mathrm{CO}$ in the core of dense clouds is, owing to the self-shielding, substantially larger than that of ${ }^{13} \mathrm{CO}$, the higher photodissociation rates of the latter molecule compete with the ion-molecule exchange (van Dishoeck and Black (1988)). It appears, therefore, that the fractionation process could be effective only in the outer regions of the dense clouds.

The study of Langer et al. (1989) mentioned above shows that the ${ }^{13} \mathrm{C} /{ }^{12} \mathrm{C}$ ratio varies only slightly with time from $10^{5}$ to $10^{6}$ years and remains constant after that. The lowest value of this ratio (about 0.7 of the "true" nominal value) is reached in a very early stage of the (modelled) cloud, between $10^{5}$ and $5 \times 10^{5}$ years. The other, less abundant carbon-bearing species in this stage have isotopic ratios larger by a factor of 1.3 than the nominal value, and after $5 \times 10^{5}$ years, these ratios increase rapidly by a factor of 1.5 to 3 . However, such a significant variation is strongly model-dependent. Nevertheless, if the sites of condensation of cometary material were in the outer regions of the protoplanetary nebula, the fractionation may have led to redistribution of the isotopic ratio among parent molecules in the cometary volatiles.

The refractory material may have a different history than the volatile compounds. As was mentioned earlier in this review, the data obtained in in situ experiments for Comet Halley exhibit large variations in the 12- and 13-a.m.u signals that cannot be directly interpreted as being the ${ }^{12} \mathrm{C} /{ }^{13} \mathrm{C}$ ratio variations. It must be noted, however that in spite of the fact that the bulk ratio of these isotopes in the dust particles is most likely the same as the terrestrial ratio or even larger, the very small fragments may exhibit a substantial enrichment either in carbon 12 or 13 . Assuming that the carbonaceous meteorites are related to cometary material, the isotopic anomalies in the inclusions buried in more complex cometary dust should not be surprising at all. (See also the distribution of the carbon isotopic ratios in various astronomical objects demonstrated in Figure 2.) Although the carbon isotope ratio in meteoric graphite is almost the same as the terrestrial ratio, a low ratio of ${ }^{12} \mathrm{C} /{ }^{13} \mathrm{C}=42$ was found (Swart et al. (1983)) in the carbon phase in primitive carbonaceous meteorites, and even lower values, around 10 to 20, were reported (Pillinger (1987); see also the review paper by Jessberger in this volume). The origin of these carbon-13-rich meteoric inclusions can be sought in red giants' atmospheres, where a low ${ }^{12} \mathrm{C} /{ }^{13} \mathrm{C}$ ratio (mostly 5 to 20 in oxygen-rich stars, and in a very wide range, from 3 to 100 , in carbonrich stars) is a well-established observational fact and is considered to be evidence for sprocess nucleosynthesis. Other sources can include ejecta from novae, but in such a case, 


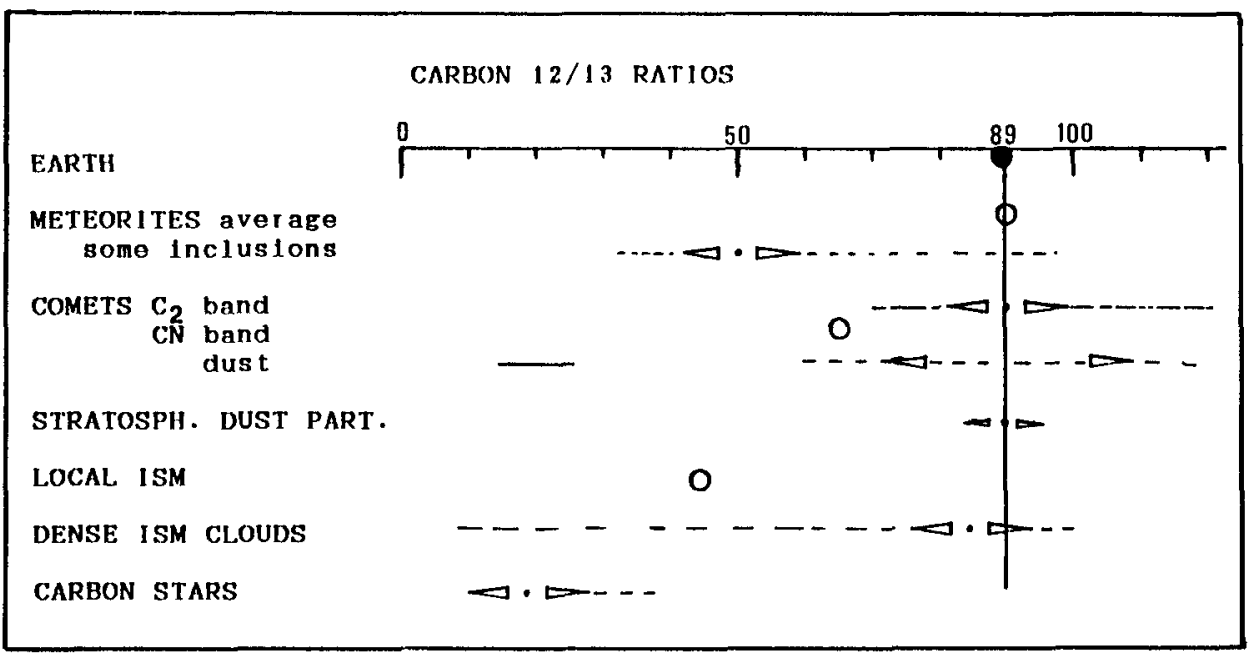

Figure 2. The ratio of stable carbon isotopes 12 and 13 in different astronomical objects compared with the terrestrial value of 89 .

the enrichment by carbon 13 should be accompanied by enrichment in nitrogen 14 . Such an effect has been found only in samples of the Allende and Murichison meteorites, where the ratio is about 400 instead of the terrestrial ratio of 272 . This, however, could also be evidence of supernovae origin. For the cometary dust, there are no conclusive data about nitrogen isotopic ratios, and the $12 / 13$ ion intensity ratio plotted versus oxygen $16 /$ nitrogen 14 shows no correlation (for details, see Jessberger (1990)). Since the supernovae ejecta, in contrast to the ejecta of red giants and novae, may contain - as a consequence of $\mathrm{He}$ burning - an enhanced abundance of carbon 12 , an extremely high value of the ${ }^{12} \mathrm{C} /{ }^{13} \mathrm{C}$ ratio in some dust samples cannot be excluded.

The interstellar origin of the submicrometer-sized dust particles as building blocks for composite refractory low-density dust grains in comets is an almost commonly accepted working concept and does not contradict the scenario of the later formation of comets in the protosolar nebula, which contains much older refractory particles with the interstellar isotopic signatures.

\section{Heavier Elements}

\subsection{NITROGEN $14 / 15$}

The only information about the ${ }^{14} \mathrm{~N} / 15 \mathrm{~N}$ ratio was obtained as a byproduct of the $\mathrm{CN}$ violet band analysis of Comet Halley used to find the carbon isotope ratio (Wyckoff $e t$ al. (1989)). If the solar system ratio of nitrogen $14 / 15=272$ is assumed, then the predicted intensity of $\mathrm{C}^{15} \mathrm{~N}$ features in the $\mathrm{CN}$ band is only about 0.25 the intensity of the 
${ }^{13} \mathrm{CN}$ lines. Since Wyckoff et al. (1989) found no evidence of the $\mathrm{C}^{15} \mathrm{~N}$ emissions, they used the noise level of the observed spectrum to conclude that the $2 \sigma$ lower limit for the nitrogen isotopic ratio is about 200 . Both the terrestrial and interstellar $(\sim 400)$ ratios are significantly larger than this lower limit, indicating that this spectrometric technique does not have the sensitivity to place stronger constraints on the nitrogen isotopic ratio. No conclusive data exist about this ratio for dust particles.

\subsection{OXYGEN $18 / 16$}

The ratios of $18 \mathrm{O} / 16 \mathrm{O}$ were determined for gas and dust constituents of Comet Halley. From data provided by the neutral gas spectrometer on the Giotto spacecraft, Eberhardt et al. (1987) found Halley's $18 \mathrm{O}$ abundance to be identical with the terrestrial one, with the ratio of ${ }^{18} \mathrm{O} / 16 \mathrm{O}$ being $0.0023 \pm 0.006$. The same can be concluded from in situ mass spectrometry of the dust, where this ratio was found to be in the range of 0.0017 to 0.0022 (Solc et al. (1987)).

\subsection{MAGNESIUM $24 / 25 / 26$}

Magnesium is the only element for which ratios of more than two stable isotopes can be measured by the mass spectroscopy in the dust of Comet Halley (Solc et al. (1987), Jessberger (1990), and references therein). The intensity of the 26/25-a.m.u. signals, which is interpreted as being the magnesium $26 / 25$ ratio, varies from 0.5 to 1.7 , and, within error limits, is the same as the terrestrial ratio (which is 1.2). Signals corresponding to 24 a.m.u. vary with respect to the 25-a.m.u signal by factors ranging from 3 to 100 , irrespective of the height at mass 25 , but this may be a contamination effect by ionized carbon molecules. There is obviously no magnesium 26 excess of more than a factor of 2 . However, the marginal excess of this isotope up to 1.15 found in meteorites and attributed to extinct unstable aluminum 26 is too small to be detected.

\subsection{OTHER HEAVY ELEMENTS}

In some reports from various mass spectrometry experiments on the VEGA and Giotto spacecraft, isotopic ratios of stable isotopes of sulfur and iron were given and were found to be consistent with terrestrial values. The ratios for chlorine 35/37 published by Solc et al. (1987) are almost certainly indicative of terrestrial contamination of the PUMA experiment and do not represent a measurement of cometary isotopic abundance.

The sulfur 34/32 ratio for the gas component in the atmosphere of Comet Halley, obtained from the neutral mass spectrometer (NMS) carried by the Giotto spacecraft, was reported as being identical to the terrestrial ratio, within $\pm 25 \%$ (Krankowsky et al. (1986)). However, the large variety of molecular species detected by this experiment makes such a finding uncertain.

The results for the ${ }^{34} \mathrm{~S} /{ }^{32} \mathrm{~S}$ and ${ }^{56} \mathrm{Fe} /{ }^{54} \mathrm{Fe}$ ratios seem accurate and could be accepted as an indicator that the cross isotopic ratios of heavy elements in the dust component of Comet Halley are identical with those found in the solar system. Further, but indirect, information about the isotopic ratio of heavy elements can be inferred from the analysis of stratospheric dust particles, if these particles are regarded as being of cometary origin 
(see, for instance, Geiss (1988)). It is evident that there are no significant deviations from the terrestrial ratios, and the results from in situ measurements can only support this fact.

\section{Conclusions}

Abundances of the stable isotopes of elements heavier than $\mathrm{C}$, including $\mathrm{O}$ and $\mathrm{S}$ in the gas phase and in the solids of comets, appear to be identical with the isotopic ratios in the solar system. It can be assumed that the same holds true for $\mathrm{Mg}, \mathrm{Si}, \mathrm{Ca}$, and $\mathrm{Fe}$ in solids. No conclusions can be drawn for the nitrogen 14/15 ratio.

The stable isotopes of magnesium, i.e., ${ }^{24} \mathrm{Mg},{ }^{25} \mathrm{Mg}$, and ${ }^{26} \mathrm{Mg}$, according to in situ measurements, show large variations in the 24/25 ratio. The 25/26 ratio seems to be the same as the terrestrial ratio, and an excess of magnesium 26 (which may partly be due to unstable aluminum 26) is probably not present. The variation of the $24 / 25$ ratio is due either to artifacts of the experiment or to contamination by molecular species.

The deuterium-to-hydrogen ratio in cometary ices covers the range of $\mathrm{D} / \mathrm{H}$ in the outer planets and in Titan, and the $\mathrm{D}$ abundance is enhanced by a factor of 10 relative to the assumed value of the $\mathrm{D} / \mathrm{H}$ ratio in the protosolar nebula. A much larger enhancement, however, cannot be excluded for other condensates in cometary nuclei. One explanationperhaps the most sensible - of such an enrichment is the ion-molecule reaction at low temperatures in a dense gas phase environment.

Although ${ }^{12} \mathrm{C} /{ }^{13} \mathrm{C}$ ratios derived from the ${ }^{12} \mathrm{C}^{13} \mathrm{C} \Delta \mathrm{v}=+1$ band tend to be, on the average, the same as the terrestrial ones, the recent very precise results for Comet Halley obtained from high-resolution spectra of the violet $\mathrm{CN}(0,0)$ band provide a significantly lower value of $65 \pm 9$ for this ratio. This corresponds to the carbon isotope ratio in the local interstellar medium. However, it is premature to conclude that the similarity between the cometary and interstellar data is evidence for an interstellar origin of comets. If the differences between the isotopic ratios for molecular carbon and $\mathrm{CN}$ are real, then they are indicative of chemical fractionation. These differences may be an inheritance transmitted from the parent molecules with different fractionation histories.

Fractionation of ${ }^{13} \mathrm{C}$ can occur via the ion-molecule reaction, which may lead to enrichment in CO, but it will be efficient only in an environment with temperatures of 10 to $30 \mathrm{~K}$ in the periphery of a dense cloud. If the precursors of $\mathrm{CN}$ molecules are " $\mathrm{CO}$ related" and those of $\mathrm{C}$ are not, then the $\mathrm{CN}$ could be significantly enriched by carbon 13 in contrast to $\mathrm{C}_{2}$, which may be depleted in the abundance of this isotope.

The mass spectrometry of ions evaporated from dust particles that have masses of 12 and 13 a.m.u. (and that may be attributed to carbon 12 and 13) in the impact mass spectrometer provides results with great variation in the ratios of these isotopes. The values tend to accumulate in two groups: most of the values are around the solar system value (89), but the smaller group of values shows a tendency to cluster around a value of about 20 . This very low value, however, is most likely a contamination effect, caused by ionized molecules of $\mathrm{CH}$, which would be a source of mass ambiguity. Therefore, in the refractory material, the dominant value of the carbon $12 / 13$ ratio is most likely the same as the terrestrial one (or perhaps somewhat higher). However, the anomalous low or high ratios (originating, for instance, in the atmospheres of red giant stars, novae, or supernovae) cannot be excluded in inclusions buried in the matrix of individual dust grains. 


\section{Acknowledgments}

I thank Dr. T. Encrenaz and an anonymous referee for many useful suggestions, which have substantially improved the final version of this paper.

\section{References}

A'Hearn, M.F., Schleicher, D.G., and West, R.A. (1985). "Emission by OD in comets." Astrophys. J. 297, 826.

Allamandola, L.J., Sandford, S.A., and Wopenka, B. (1987). "Interstellar polycyclic aromatic hydrocarbons and carbon in interplanetary dust particles and meteorites." Science 237, 56.

Anders, E. (1987). "Local and exotic components of primitive meteorites and their origin." Phil. Trans. R. Soc. London, A 323, 287.

Audouze, J. (1977). "Importance of CNO isotopes in astrophysics." In CNO Isotopes in Astrophysics, J. Audouze et al. (eds.), D. Reidel, Dordrecht, p. 155.

Bastien, P., Bartla, W., Henkel, C., Paulus, T., Walmsley, C.M., and Wilson, T.L. (1985). "Formaldehyde observation in OMC-1." Astron. Astrophys. 149, 86.

Beer, R., and Taylor, F.W. (1973). "The D/H and C/H ratios in Jupiter." Astrophys. J. $219,763$.

Black, D.C. (1973). "Deuterium in the early solar system." Icarus 19, 154.

Brown, P.D., and Millar, T.J. (1989a). "Models of the gas-grain interaction deuterium chemistry." Month. Not. RAS 237, 661.

Brown, P.D., and Millar, T.J. (1989b). "Grain-surface formation of multi-deuterated molecules." Month. Not. RAS 240, 25.

Brown, R.D., and Rice, E.H.N. (1986). "Galactochemistry II, interstellar deuterium chemistry." Month. Not. RAS 223, 429.

Combes, F., Falgarone, E., Guibert, J., and Nguyen-Q-Rieu (1980). "CO observations of interstellar clouds: Isotopic ratios." Astron. Astrophys. 90, 88.

Danks, A.C., Lambert, D.L., and Arpigny, C. (1974). "The ${ }^{12} \mathrm{C} /{ }^{13} \mathrm{C}$ ratio in comet Kohoutek (1973 f)." Astrophys. 194, 745.

de Bergh, C., Lutz, B.L., Owen, T., Brault, J., and Chauville, J. (1986). "Monodeuterated methane in the outer solar system. II. Its detection on Uranus at $1.6 \mu \mathrm{m}$." Astrophys. J. 311, 501.

de Bergh, C., Lutz, B.L., and Owen, T. (1988). "Monodeuterated methane in the outer solar system. III. Its abundance on Titan." Astrophys. J. 329, 951.

de Bergh, C., Lutz, B.L., and Owen, T. (1989). "Monodeuterated methane in the outer solar system. IV. Its detection and abundance on Neptune." Astrophys. J., in press.

Eberhardt, P., Krankowsky, D., Schulte, W., Dolder, U., Laemmerzahl, P., Bertheier, J.J., Woweries, J., Stubbemann, U., Hodges, R.R., Hoffmann, J.H., and Illiano, J.M. (1987). "The D/H ratio in water from comet P/Halley." Astron. Astrophys. 187, 435.

Encrenaz, T. (1984). "Isotopic ratios in comets." In Isotopic Ratios in the Solar System, Cepadeus-Edition, Toulouse, p. 173. 
Gautier, D., and Owen, T. (1983). "Cosmological implication of elemental and isotopic abundances in atmospheres of Jovian planets." Nature 302, 215.

Geiss, J. (1988). "Composition in Halley's comet: Clues to origin and history of cometary matter." In Reviews of Modern Astronomy 1, G. Klare (ed.), SpringerVerlag, Heidelberg, p. 1.

Geiss, J., and Reeves, H. (1972). "Cosmic and solar system abundance of deuterium and helium 3." Astron. Astrophys. 18, 126.

Geiss, J., and Reeves, H. (1981). "Deuterium in the solar system." Astron. Astrophys. 93, 189.

Gerin, J., Combez, F., Encrenaz, P., Linke, R., Destombes, J.L., and Demuynck, C. (1984). "Detection of ${ }^{13} \mathrm{CN}$ in three galactic sources." Astron. Astrophys. 136, L17.

Grinspoon, D., and Lewis, J.S. (1987). "Deuterium fractionation in the presolar nebula." Icarus $78,430$.

Hawkins, I., and Jura, M. (1987). "The ${ }^{12} \mathrm{C} /{ }^{13} \mathrm{C}$ isotope ratio of the interstellar medium in the neighborhood of the sun." Astrophys. J. 317, 926.

Herbst, E. (1988). "Interstellar molecular formation process." In Reviews of Modern Astronomy 1, G. Klare (ed.), Springer-Verlag, Heidelberg, p. 114.

Hubbart, W.B., and McFarlane, J.J. (1980). "Theoretical prediction of deuterium abundance in Jovian planets." Icarus 44, 676.

Ip, W.-H. (1985). "Condensations and agglomeration of cometary ice: The HDO/HO ratios as traces." In Ices in the Solar System, J. Klinger et al. (eds.), D. Reidel, Dordrecht, p. 389.

Jessberger, E.K. (1990). "Chemical properties of cometary dust and a note on carbon isotopes." In this volume.

Jessberger, E.K., Kissel, J., Fechtig, H., and Krueger, F.R. (1986). "On average chemical composition of cometary dust." ESA Spec. Publ. 249, 27.

Krankowsky, D., Eberhardt, P., Dolder, U., Schulte, W., Laemmerzahl, P., Hoffmann, J.H., Hodges, R.R., Berthelier, J.J., and Illiano, J.M. (1986). "In-situ gas and ion measurements at Comet Halley." Nature 321, 326.

Krishna Swamy, K.S. (1987). "Study of isotope features of Swan bands in comets." Astron. Astrophys. 187, 388.

Lambert, D.L., and Danks, A.C. (1983). "High resolution spectra of $\mathrm{C}_{2}$ Swan bands from comet West." Astrophys. J. 268, 428.

Langer, W.D., and Graedel, T.E. (1989). "Ion-molecule chemistry of dense clouds." Astrophys. J. Suppl. 69, 241.

Langer, W.D., Graedel, T.E., Frerking, M.A., and Armentroud, J. (1984). "Carbon and oxygen isotope fractionation in dense interstellar clouds." Astrophys. J. 277, 587.

Lutz, B.L., de Bergh, C., and Maillard, J.P. (1983). "Monodeuterated methane in outer solar system I." Astrophys. J. 273, 397.

Lutz, B.L., Owen, T., and de Bergh, C. (1989). "Deuterium enrichment in primitive ices of the solar nebula." Lowell Observatory Preprints.

McKeegan, K.D., Walker, R.M., and Zinner, E. (1985). "Ion microprobe isotopic measurements of individual interplanetary dust particles." Geochim. Cosmochim. Acta, 49.

Millar, T.J., Bennet, A., and Herbst, E. (1989). "Deuterium fractionation in dense interstellar clouds." Astrophys. J. 340, 906. 
Owen, T., Lutz, B.L., and de Bergh, C. (1986). "Deuterium in the outer solar system." Nature 320, 224.

Pasachoff, J.M., and Vidal-Madjar, A. (1989). "The need to observe the distribution of interstellar deuterium." Comments in Astrophysics 14, 61.

Penzias, A.A. (1983). "Measurement of isotopic abundances in interstellar clouds." In Interstellar Molecules, B.H. Andrew (ed.), D. Reidel, Dordrecht, p. 397.

Pillinger, C.T. (1987). "Stable isotope measurements of meteorites and cosmic dust grains." Phil. Trans. R. Soc. London A 323, 313.

Robert, F., Melivat, L., and Javoy, M. (1979). "Water and deuterium content in the Chainpur and Orgueil meteorites." Nature 289, 785.

Sarmiento, A., and Peimebert, M. (1985). "Novae and galactic chemical evolution." Rev. Mexicana Astr. Astrophys. 11, 73.

Singh, P.D., and Dalgarno, A. (1987). "Photodissociation lifetimes of $\mathrm{CH}$ and CD radicals in comets." ESA Spec. Publ. 278, 177.

Smith, D. (1987). "Interstellar molecules." Phil. Trans. R. Soc. London A 323, 269.

Smith, H.W., Schempp, W.V., and Baines, K.H. (1989). "D/H for Uranus and Neptune." Astrophys. J. 336, 976.

Solc, M., Vanysek, V., and Kissel, J. (1987). "Carbon-isotope ratio in PUMA 1 spectra of P/Halley." Astron. Astrophys. 187, 385.

Stawikowski, A., and Greenstein, J.L. (1964). "Isotope ratio ${ }^{12} \mathrm{C} /{ }^{13} \mathrm{C}$ in a comet." Astrophys. J. 140, 1280.

Swart, P.K., Grady, M.M., Pillinger, C.T., Lewis, R.S., and Anders, E. (1983). "Interstellar carbon in meteorites." Science 200, 406.

Tielens, A.G.G.M. (1983). "Surface chemistry of deuterated molecules." Astron. Astrophys. 119, 177.

van Dishoeck, E.F., and Black, J.H. (1988). "Diffuse cloud chemistry." In Rate Coefficients in Astrochemistry, T.J. Millar and D.A. Williams (eds.), Kluwer Academic Press, Dordrecht, p. 209.

Vanysek, V. (1977). "Carbon isotope ratio in comets and interstellar matter." In Comets, Asteroids and Meteorites, A.H. Delsemme (ed.), University of Toledo Press, Toledo, p. 499.

Vanysek, V., and Rahe, J. (1978). "The carbon isotopes in comets." Moon and Planets $18,441$.

Vanysek, V., and Vanysek, P. (1985). "Prediction of deuterium abundance in comets." Icarus 61, 57.

Vidal-Madjar, A. (1983). "Interstellar helium and deuterium." In Diffuse Matter in Galaxies, J. Audouze et al. (eds.), D. Reidel, Dordrecht, p. 57.

Wyckoff, S., Lindholm, E., Wehinger, P.A., Peterson, B.A., Zucconi, J.M., and Festou, M.C. (1989). "The ${ }^{12} \mathrm{C} /{ }^{13} \mathrm{C}$ abundance ratio in Comet Halley." Astrophys. J. 339, 488.

Yang, J., and Epstein, S. (1983). "On the origin and composition of hydrogen and carbon in meteorites." Geochim. Cosmochim. Acta 47, 2199.

Zucconi, J.M., and Festou, M.C. (1986). "The isotopes of CN in comets." Astron. Astrophys. 158, 382. 